\title{
Cultura de segurança do paciente em uma maternidade de risco habitual
}

\section{Patient safety culture in a low-risk maternity hospital}

Fernanda de Jesus Santos ${ }^{1}$, Hertaline Menezes do Nascimento ${ }^{1}$, José Marcos de Jesus Santos ${ }^{1}$, Jéssica Oliveira da Cunha ${ }^{1}$ Jamille Carolina Silva Santos ${ }^{1}$, Juliana de Araújo Pena ${ }^{1}$

DOI: https://dx.doi.org/10.7322/abcshs.v44i1.1066

\section{RESUMO}

Introdução: A preocupação com a segurança dos pacientes tornou-se prioritária nas últimas décadas, pois se trata de um princípio básico e importante requisito para garantia da qualidade do cuidado. Objetivo: Avaliar a cultura de segurança dos pacientes na perspectiva dos profissionais de saúde que trabalham em uma maternidade de risco habitual. Métodos: Trata-se de um estudo transversal e quantitativo, com abordagem descritiva, realizado entre os meses de janeiro e março de 2017 com 21 profissionais de saúde atuantes em uma maternidade filantrópica do município de Lagarto, Sergipe, Brasil. As informações foram obtidas por meio do Safety Attitudes Questionnaire (SAQ), que avalia em escores as percepções de profissionais em relação à segurança do paciente em seis grandes domínios. Ressaltase que é considerado escore positivo qualquer domínio com média $\geq 75$ pontos. Resultados: Os domínios Clima de Trabalho em Equipe (escore=79,1), Satisfação no Trabalho (escore=81,4), Percepção do stress (escore=76,7) e Condições de Trabalho (escore $=78,8$ ) apresentaram-se com uma avaliação positiva no escore do instrumento utilizado (média $\geq 75$ pontos). Somente os domínios Clima de Segurança (escore=73,9) e Percepção da Gerência (escore $=59,6$ ) se mostraram com pontuação abaixo do escore esperado para a cultura de segurança do paciente. Conclusão: De modo geral, foi evidenciada uma cultura positiva de segurança do paciente na maternidade estudada, com necessidade de pequenas adequações no comprometimento organizacional e gerencial do serviço.

Palavras-chave: segurança do paciente; qualidade da assistência à saúde; maternidades; atenção à saúde.

\begin{abstract}
Introduction: Concern with patient safety has become a priority in the last decades, since it is a basic principle and an important requirement to guarantee the quality of care. Objective: To evaluate the patient safety culture from the perspective of the health professionals who work in a low-risk maternity hospital. Methods: A cross-sectional and quantitative study, with descriptive approach, carried out between January and March 2017 with 21 health professionals working in a nos-profit maternity hospital in the town of Lagarto, Sergipe, Brazil. The information was obtained through the Safety Attitudes Questionnaire (SAQ), which evaluates in scores the attitudes and professionals' perceptions regarding patient safety in six domains. Any domain with mean $\geq 75$ points is considered a positive score. Results: The domains Teamwork Climate (score=79.1), Job Satisfaction (score=81.4), Stress Recognition (score=76.7) and Working Conditions (score=78.8) scored above 75 points. Only the domains Safety Climate (score=73.9) and Perception of Management (score=59.6) were below the expected scored for the patient safety culture. Conclusion: In general, a positive patient safety culture was found in the maternity hospital studied, with the necessity of organizational adaptations.
\end{abstract}

Keywords: patient safety; quality of health care; hospitals, maternity; health care. 


\section{INTRODUÇÃO}

A preocupação com a segurança dos pacientes tornou-se prioritária nas últimas décadas, pois se trata de um princípio básico e um requisito para a garantia da qualidade do cuidado. Entende-se por segurança do paciente o ato de prevenir ou reduzir os riscos de danos decorrentes da assistência médico-hospitalar ${ }^{1}$.

Sabe-se que embora os profissionais de saúde possuam a premissa de proporcionar a melhor assistência aos seus pacientes, a ocorrência de erros é uma realidade, e quando ocorrem, uma das principais consequências é o agravamento imediato ou tardio do quadro clínico dos enfermos envolvidos ${ }^{2,3}$. Trata-se de uma problemática mundial, com resultados negativos inclusive em países como Estados Unidos ${ }^{4}$ e República da Albânia ${ }^{5}$.

No contexto hospitalar, pressupõe-se que a segurança nos procedimentos maternos e neonatais é de uma importância ainda maior, tendo em vista as diversas possibilidades de eventos adversos neste processo assistencial ${ }^{6}$. No Brasil, ocorrem aproximadamente três milhões de nascimentos a cada $\mathrm{ano}^{7}$, e merece destacar que em casos de procedimentos obstétricos imprudentes, o sofrimento de afecções no período perinatal poderá resultar em novas internações hospitalares e/ou mortes ${ }^{8}$. Destaca-se que, na época dessa pesquisa, não havia ainda nenhum outro estudo nacional avaliando a cultura de segurança do paciente em uma maternidade de risco habitual.

Dessa forma, ao considerar que a cultura de segurança do paciente requer o compartilhamento de valores e atitudes que visam à redução dos possíveis danos assistenciais entre os profissionais e serviços de saúde ${ }^{1}$, o objetivo desse estudo foi avaliar a cultura de segurança dos pacientes na perspectiva dos profissionais de saúde que trabalham em uma maternidade de risco habitual de um município do Nordeste brasileiro.

\section{MÉTODOS}

Trata-se de um estudo transversal e quantitativo, com abordagem descritiva, realizado entre os meses de janeiro e março de 2017 com 21 profissionais de saúde atuantes em uma maternidade filantrópica do município de Lagarto, Sergipe, Brasil. A maternidade estudada possui quatro leitos particulares, seis leitos de pré-parto e 31 leitos de alojamento conjunto. Atende partos com financiamento público e/ou privado de parturientes lagartenses e outras provenientes de cidades circunvizinhas.

Segundo dados da direção da instituição, existiam 40 profissionais de saúde da área assistencial atuantes na maternidade à época da pesquisa. Destes, somente 22 eram considerados elegíveis por atenderem aos critérios de inclusão do estudo (cinco enfermeiros, 13 técnicos de enfermagem, um médico obstetra/pediatra e dois nutricionistas). Destaca-se que, apesar de esta ser uma população pequena, optou-se ainda por realizar cálculo amostral usando a fórmula de Barbetta ${ }^{9}$ e considerando um nível de confiança de
95\% e de erro amostral de 5\%, o que resultou em 21 sujeitos entrevistados. Utilizou-se a amostragem aleatória simples para alcance da amostra (foi sorteado, para exclusão, um profissional de saúde dentre os 22 elegíveis).

Como critério de inclusão adotou-se: trabalhar no setor atual por um período mínimo de um mês e com carga horária semanal de no mínimo 24 horas. Justifica-se a opção de utilizar como critério de inclusão a atuação profissional de apenas um mês no setor atual por se tratar de uma unidade com fácil adaptação e/ou de rápido conhecimento estrutural e funcional.

A coleta dos dados ocorreu por meio do Safety Attitudes Questionnaire (SAQ), criado em 2006 para avaliar as percepções de profissionais em relação a questões de segurança do paciente. Esse instrumento passou por um processo de adaptação transcultural para o Brasil (alfa de 0.89) e possui 41 questões que englobam seis domínios e ainda uma segunda parte para coleta de dados socioeconômicos dos profissionais. As respostas a cada uma das questões seguem uma escala Likert de cinco pontos: discordo totalmente, discordo um pouco, neutro, concordo um pouco, concordo totalmente e não se aplica ${ }^{10}$.

Destaca-se que devido ao fato de ser um instrumento de coleta padrão e disponível para acesso público, os autores deste estudo não consideraram ser necessária à solicitação de permissão para sua utilização. Ademais, optou-se por incluir apenas 36 itens devido às especificidades do serviço escolhido para a coleta dos dados, haja vista que as demais não se aplicariam à forma de funcionamento da Instituição. Pontua-se que isso não interferiu na realização dos cálculos do escore de cada domínio avaliado.

A avaliação da percepção de segurança ocorreu por meio destes seis grandes domínios: 1) Clima de Trabalho em Equipe (itens 1 a 6); 2) Clima de Segurança (itens 7 a 13); 3) Satisfação no Trabalho (itens 15 a 19); 4) Percepção do stress (itens 20 a 23); 5) Percepção da Gerência (itens 24 a 29) e 6) Condições de Trabalho (itens 30 a 33). Ressalta-se que os itens 14, 34, 35 e 36 não fazem parte de nenhum domínio no instrumento original ${ }^{2}$.

O escore do SAQ varia de 0 (pior percepção de segurança do paciente por parte dos profissionais de saúde) a 100 pontos (melhor percepção). Considera-se valor positivo quando a média do escore é igual ou maior que 75. A pontuação se deu da seguinte forma: $0=$ discorda totalmente, $25=$ discorda parcialmente, $50=$ neutro, $75=$ concorda parcialmente, $100=$ concorda totalmente e $\mathrm{X}=$ não se aplica. $\mathrm{O}$ cálculo do escore dos domínios foi realizado a partir da média aritmética dos seus itens. Destaca-se que os itens 2, 11 e 36 do SAQ são referentes a questões reversas, ou seja, considerou-se "concordo totalmente" (100) quando o participante assinalou "eu discordo totalmente" (0), e assim por diante.

Para realização da análise estatística do tipo descritiva foi utilizada a técnica univariada para obtenção dos valores das frequências absoluta e relativa entre as variáveis nominais. Também foram calculados os valores das medidas de tendência central e do 
desvio padrão das variáveis numéricas. O programa utilizado foi o IBM ${ }^{\circledR}$ SPSS - Statistical Package for the Social Sciences 20.0 Mac (SPSS 20.0 Mac, SPSS Inc., Chicago, Illinois, EUA).

Obteve-se a aprovação deste estudo pelo Comitê de Ética em Pesquisa da Universidade Federal de Sergipe, sob parecer $\mathrm{n}^{\circ}$ 1.313.955, com o seguinte CAAE: 48296315.6.0000.5546. Todos os cuidados foram adotados visando garantir o sigilo e confidencialidade das informações, conforme Resolução no 466/2012 do Conselho Nacional de Saúde.

\section{RESULTADOS}

A média de idade dos participantes era de 34,7 anos (desvio padrão=10), sendo 27 a mínima e 52 a máxima. A maioria era mulher $(95,2 \% ; n=20)$, técnica de enfermagem $(61,9 \% ; n=13)$ ou enfermeira $(23,8 \% ; n=5)$, trabalhava no setor do pré-parto $(57,1 \% ; n=12)$ ou pós-parto $(38,1 \% ; n=8)$ e possuía um tempo de atuação profissional inferior a cinco anos à época da pesquisa $(61,9 \% ; n=13)$ (Tabela 1$)$.

O escore geral do Safety Attitudes Questionnaire (SAQ) foi de 74,2 pontos na maternidade estudada. Os domínios Clima de Trabalho em Equipe (escore=79,1), Satisfação no Trabalho (escore=81,4), Percepção do stress (escore=76,7) e Condições de Trabalho (escore $=78,8$ ) obtiveram média maior que 75 pontos, isto é, apresentaram uma avaliação positiva no escore do instrumento utilizado. Os domínios Clima de Segurança (escore=73,9) e Percepção da Gerência (escore=59,6), assim como o escore geral, também se mostraram abaixo do esperado para a garantia da cultura de segurança do paciente (Tabela 2 ).

Quanto aos itens do domínio Clima de Trabalho em Equipe, verificou-se que 95,2\% $(n=20)$ dos profissionais têm o apoio de outros membros da equipe e que as discordâncias são resolvidas de modo apropriado no local de trabalho, bem como 90,5\% ( $\mathrm{n}=19)$ referiram que fazem perguntas quando não entendem alguma coisa e que o trabalho em equipe realmente acontece. Quanto ao Clima de Segurança, 95,2\% $(n=20)$ disseram informar qualquer preocupação e $85,7 \%(n=18)$ acreditam que os erros da equipe de trabalho são analisados adequadamente e que possuem conhecimento suficiente para o manejo correto de eventuais problemas de segurança do paciente na maternidade estudada (Tabela 3).

Em relação ao domínio Satisfação no Trabalho, 90,5\% (n=19) dos profissionais concordam que trabalham em um bom lugar e que se sentem orgulhosos da área de atuação escolhida. Ademais, $85,7 \%(\mathrm{n}=18)$ gostam do trabalho atual e se sentem como sendo uma grande família neste local. No domínio Percepção do stress, $95,2 \%(n=20)$ referiram que são menos eficientes no trabalho quando estão cansados e $85,7 \%(n=18)$ acreditam que a carga de trabalho excessiva prejudica seu desempenho profissional (Tabela 4).

A maioria dos profissionais referiu receber informações adequadas e oportunas sobre eventos que podem afetar o funcionamento da unidade $(90,5 \% ; n=19)$ e que a administração atual está fazendo um bom trabalho $(76,2 \% ; n=16)$. No domínio Condições de Trabalho, 85,7\% (n=18) referiram vivenciar uma boa colaboração com os enfermeiros e $81 \%(n=17)$ concordam que a unidade faz um bom trabalho no treinamento de novos membros da equipe (Tabela 5).

\section{DISCUSSÃO}

Embora o escore geral do Safety Attitudes Questionnaire (SAQ) na maternidade estudada tenha se mostrado um pouco inferior ao limite aceitável, a avaliação individualizada dos domínios evidenciou uma cultura positiva de segurança do paciente, pois quatro

Tabela 1: Resultados descritivos das variáveis socioeconômicas de 21 profissionais de saúde de uma maternidade de risco habitual. Lagarto, Sergipe, Brasil, 2017.

\begin{tabular}{|l|c|c|}
\hline Variáveis socioeconômicas & $\mathbf{N}^{*}$ & $\%{ }^{\star *}$ \\
\hline Faixa etária & & 19,1 \\
\hline $20-30$ & 14 & 66,6 \\
\hline $31-40$ & 2 & 9,5 \\
\hline $41-50$ & 1 & 4,8 \\
\hline 51 ou mais & & \\
\hline Sexo & 20 & 95,2 \\
\hline Feminino & 1 & 4,8 \\
\hline Masculino & & \\
\hline Categoria profissional & 5 & 23,8 \\
\hline Enfermeiro & 13 & 61,9 \\
\hline Técnico de Enfermagem & 1 & 4,8 \\
\hline Médico obstetra ou pediatra & 2 & 9,5 \\
\hline Nutricionista & & \\
\hline Setor de trabalho na instituição & 1 & 4,8 \\
\hline Acolhimento & 12 & 57,1 \\
\hline Pré-parto & 8 & 38,1 \\
\hline Pós-parto & & \\
\hline Tempo de atuação profissional & 13 & 61,9 \\
\hline 0 a 5 anos & 8 & 38,1 \\
\hline 6 a 10 anos & & \\
\hline * (frequencia & & \\
\hline
\end{tabular}

* $N$ (frequência absoluta), ** \% (frequência relativa).

Tabela 2: Resultados descritivos dos domínios do Questionário de Atitude de Segurança (SAQ). Lagarto, Sergipe, Brasil, 2017.

\begin{tabular}{|l|c|c|c|}
\hline Domínios & Média & Desvio Padrão & Mediana \\
\hline Clima de Trabalho em Equipe & $79,1^{*}$ & 23,3 & 79,1 \\
\hline Clima de Segurança & 73,9 & 25,1 & 85,7 \\
\hline Satisfação no Trabalho & $81,4^{*}$ & 33,1 & 95,0 \\
\hline Percepção do stress & $76,7^{*}$ & 27,8 & 87,5 \\
\hline Percepção da Gerência & 59,6 & 33,2 & 70,8 \\
\hline Condições de Trabalho & $78,8^{\star}$ & 30,8 & 93,7 \\
\hline Itens Isolados & 70,2 & 32,4 & 83,3 \\
\hline SAQ Geral & 74,2 & 29,3 & 85,0 \\
\hline
\end{tabular}

*Avaliação positiva (média $\geq 75$ pontos) 
dos seis domínios apresentaram-se com média maior que 75 pontos no escore do instrumento utilizado. Estes resultados mostram-se melhores quando comparados a outros estudos nacionais com delineamento metodológico semelhante, cujos escores gerais foram de 61,8 pontos e também inferiores a 75 em cada um dos domínios avaliados ${ }^{11,12}$.

Os dois domínios com escore abaixo de 75 pontos, indicando uma percepção negativa do clima de segurança, foram Clima de
Segurança e Percepção da Gerência, que estão relacionados ao comprometimento organizacional e gerencial do serviço, respectivamente. No entanto, vale ressaltar que a maioria dos participantes do serviço afirmou receber informações adequadas e oportunas sobre quaisquer eventos que possam afetar o funcionamento da unidade, e que a administração atual está fazendo um bom trabalho.

No domínio Clima de Trabalho em Equipe percebeu-se a existência de apoio entre os membros das equipes e que as

Tabela 3: Distribuição das respostas por item nos domínios Clima de Trabalho em Equipe e Clima de Segurança de 21 profissionais de saúde de uma maternidade de risco habitual. Lagarto, Sergipe, Brasil, 2017.

\begin{tabular}{|c|c|c|c|}
\hline \multicolumn{4}{|l|}{ Domínios } \\
\hline Clima de Trabalho em Equipe & $\begin{array}{l}\text { Discordo* } \\
\mathrm{N}(\%)\end{array}$ & $\begin{array}{l}\text { Neutro } \\
\mathrm{N}(\%)\end{array}$ & $\begin{array}{l}\text { Concordo* } \\
\text { N (\%) }\end{array}$ \\
\hline As sugestões do enfermeiro são bem recebidas nesta área. & $0(0)$ & $3(14,3)$ & $18(85,8)$ \\
\hline Nesta área, é difícil falar abertamente se eu percebo um problema com o cuidado ao paciente. ${ }^{* *}$ & $8(38)$ & $1(4,8)$ & $12(57,1)$ \\
\hline Nesta área, as discordâncias são resolvidas de modo apropriado. & $0(0)$ & $1(4,8)$ & $20(95,2)$ \\
\hline Eu tenho o apoio que necessito de outros membros da equipe para cuidar dos pacientes. & $1(4,8)$ & $0(0)$ & $20(95,2)$ \\
\hline $\begin{array}{l}\text { É fácil para os profissionais que atuam nesta área fazerem perguntas quando existe algo que eles não } \\
\text { entendem. }\end{array}$ & $2(9,5)$ & $0(0)$ & $19(90,5)$ \\
\hline Os médicos e enfermeiros daqui trabalham juntos como uma equipe bem coordenada. & $2(9,5)$ & $0(0)$ & $19(90,5)$ \\
\hline Clima de Segurança & $\begin{array}{l}\text { Discordo* } \\
\mathrm{N}(\%)\end{array}$ & $\begin{array}{l}\text { Neutro } \\
\mathbf{N}(\%)\end{array}$ & $\begin{array}{l}\text { Concordo* } \\
\text { N (\%) }\end{array}$ \\
\hline Eu me sentiria seguro se fosse tratado aqui como paciente. & $0(0)$ & $0(0)$ & $21(100)$ \\
\hline Erros são tratados de forma apropriada nesta área. & $2(9,5)$ & $1(4,8)$ & $18(85,7)$ \\
\hline Eu conheço os meios adequados para encaminhar as questões relacionadas à segurança do paciente. & $2(9,5)$ & $1(4,8)$ & $18(85,7)$ \\
\hline Eu recebo retorno apropriado sobre meu desempenho. & $4(19)$ & $4(19)$ & $13(61,9)$ \\
\hline Nesta área, é difícil discutir sobre erros. ${ }^{* *}$ & $4(19)$ & $1(4,8)$ & $16(76,2)$ \\
\hline $\begin{array}{l}\text { Sou encorajado por meus colegas a informar qualquer preocupação que eu possa ter quanto à segurança } \\
\text { do paciente. }\end{array}$ & $1(4,8)$ & $0(0)$ & $20(95,2)$ \\
\hline A cultura nesta área torna fácil aprender com os erros dos outros. & $9(42,8)$ & $1(4,8)$ & $11(52,4)$ \\
\hline
\end{tabular}

*Parcial ou totalmente, ${ }^{* *}$ Questões reversas.

Tabela 4: Distribuição das respostas por item nos domínios Satisfação no Trabalho e Percepção do stress de 21 profissionais de saúde de uma maternidade de risco habitual. Lagarto, Sergipe, Brasil, 2017.

\begin{tabular}{|l|c|c|c|}
\hline Domínios & \multicolumn{3}{|c|}{ Respostas } \\
\hline Satisfação no Trabalho & $\begin{array}{c}\text { Discordo* } \\
\mathbf{N}(\%)\end{array}$ & $\begin{array}{c}\text { Neutro } \\
\mathbf{N}(\%)\end{array}$ & $\begin{array}{c}\text { Concordo* } \\
\mathbf{N}(\%)\end{array}$ \\
\hline Eu gosto do meu trabalho. & $2(9,5)$ & $0(0)$ & $18(85,7)$ \\
$\mathbf{N}(\%)$ \\
\hline Trabalhar aqui é como fazer parte de uma grande família. & $2(4,8)$ \\
\hline Este é um bom lugar para trabalhar. & $2(9,5)$ & $1(4,8)$ & $18(85,7)$ \\
\hline Eu me orgulho de trabalhar nesta área. & $2(9,5)$ & $0(0)$ & $19(90,5)$ \\
\hline O moral nesta área é alto. & $2(9,5)$ & $0(0)$ & $19(90,5)$ \\
\hline Percepção do stress & $6(28,5)$ & $2(9,5)$ & $12(57,2)$ \\
\hline Quando minha carga de trabalho é excessiva, meu desempenho é prejudicado. & Discordo* & $\mathbf{N e u t r o}$ & $\mathbf{1}(4,8)$ \\
\hline Eu sou menos eficiente no trabalho quando estou cansado. & $\mathbf{N}(\%)$ & $\mathbf{N}(\%)$ & $\mathbf{N}(\%)$ \\
\hline Eu tenho maior probabilidade de cometer erros em situações tensas ou hostis. & $3(14,3)$ & $0(0)$ & $18(85,7)$ \\
\hline O cansaço prejudica meu desempenho durante situações de emergência. & $0(0)$ & $1(4,8)$ & $20(95,2)$ \\
\hline
\end{tabular}

*Parcial ou totalmente, ${ }^{* *}$ Não se aplica. 
discordâncias são resolvidas de modo apropriado no local de trabalho, bem como foi referido que o trabalho em equipe de fato acontece. Estas situações positivas assemelham-se às de outro estudo $^{13}$ realizado em sete hospitais no estado do Rio Grande do Sul. Este resultado pode ser justificado pelas respostas encontradas no domínio Condições de Trabalho, no qual os participantes referiram vivenciar uma boa colaboração com os enfermeiros e concordam que a unidade faz um bom trabalho no treinamento de novos membros da equipe.

O domínio Satisfação no Trabalho obteve o maior escore positivo, fato também observado em um estudo realizado em três hospitais do estado do Ceará ${ }^{14}$. Outros estudos nacionais ${ }^{15,16}$ também evidenciaram escores positivos no domínio Satisfação no Trabalho, demonstrando que, de modo geral, os profissionais de saúde estão satisfeitos com o trabalho que desenvolvem. Ainda assim, é importante ressaltar que a insatisfação profissional pode repercutir na execução de tarefas com baixa qualidade, sendo responsável por esquecimentos, falhas e atrasos nas atividades desempenhadas e dificuldades de relacionamento com a equipe ${ }^{17}$.

Ademais, ao compararmos estes resultados com outros estudos internacionais, observa-se que os profissionais que participaram desta pesquisa estão mais satisfeitos com o seu trabalho que alguns profissionais dos Estados Unidos e Irlanda ${ }^{4,18}$. Acredita-se que a instituição é a principal responsável por este fenômeno, já que a oferta de um ambiente propício para a execução do trabalho favorece a tomada de atitudes positivas para a segurança do paciente e, consequentemente, traz satisfação ao profissional que as desenvolve. Cabe destacar que a instituição na qual o presente estudo foi realizado oferece com frequência treinamentos/ atualizações a toda equipe profissional, bem como mantém uma ambiência favorável à humanização da assistência e dispõe de insumos em quantidade suficiente para realização dos cuidados. Esse ambiente saudável impacta sobre o clima organizacional e, consequentemente, repercute na segurança do paciente.

Outro domínio com resultado positivo no presente estudo foi a Percepção do stress, relacionada à identificação dos fatores estressores que influenciam na execução do trabalho, fato também observado em um hospital público brasileiro ${ }^{15}$. Sabe-se que a dificuldade em perceber e também em lidar com fatores estressores podem resultar em erros de trabalho, redução da produtividade, sentimentos de desconforto, doença ou mau desempenho da equipe ${ }^{19}$.

A principal limitação desse estudo está relacionada ao pequeno número amostral, uma vez que isso impossibilitou a realização de análises estatísticas mais robustas e inferenciais.

Foi evidenciada uma cultura positiva de segurança do paciente na maternidade estudada, com necessidade de pequenas adequações no comprometimento organizacional e gerencial do serviço. Sabe-se que a gerência tem um papel decisivo na garantia da segurança do paciente, já que a identificação e correção de possíveis

Tabela 5: Distribuição das respostas por item nos domínios Percepção da Gerência, Condições de Trabalho e Itens Isolados de 21 profissionais de saúde de uma maternidade de risco habitual. Lagarto, Sergipe, Brasil, 2017.

\begin{tabular}{|c|c|c|c|c|}
\hline \multirow{2}{*}{$\begin{array}{l}\text { Domínios } \\
\text { Percepção da Gerência }\end{array}$} & \multicolumn{4}{|c|}{ Respostas } \\
\hline & $\begin{array}{l}\text { Discordo* } \\
\text { N (\%) }\end{array}$ & $\begin{array}{l}\text { Neutro } \\
\mathrm{N}(\%)\end{array}$ & $\begin{array}{l}\text { Concordo* } \\
\mathrm{N}(\%)\end{array}$ & $\begin{array}{l}N A^{\star *} \\
N(\%)\end{array}$ \\
\hline A administração apoia meus esforços diários. & $7(33,3)$ & $3(14,3)$ & $11(52,4)$ & - \\
\hline A administração não compromete conscientemente a segurança do paciente. & $6(28,5)$ & $2(9,5)$ & $12(57,2)$ & $1(4,8)$ \\
\hline A administração está fazendo um bom trabalho. & $3(14,3)$ & $2(9,5)$ & $16(76,2)$ & - \\
\hline Profissionais problemáticos da equipe são tratados de maneira construtiva por nossa unidade. & $6(28,6)$ & $2(9,5)$ & $13(61,9)$ & - \\
\hline $\begin{array}{l}\text { Recebo informações adequadas e oportunas sobre eventos que podem afetar meu trabalho } \\
\text { da unidade. }\end{array}$ & $2(9,5)$ & $0(0)$ & $19(90,5)$ & - \\
\hline $\begin{array}{l}\text { Nesta área, o número e a qualificação dos profissionais são suficientes para lidar com o } \\
\text { número de pacientes. }\end{array}$ & $15(71,4)$ & $1(4,8)$ & $5(23,8)$ & - \\
\hline Condições de Trabalho & $\begin{array}{l}\text { Discordo* } \\
\text { N (\%) }\end{array}$ & $\begin{array}{l}\text { Neutro } \\
\mathrm{N}(\%)\end{array}$ & \multicolumn{2}{|c|}{$\begin{array}{l}\text { Concordo* } \\
\mathrm{N}(\%)\end{array}$} \\
\hline Este hospital faz um bom trabalho no treinamento de novos membros da equipe. & $2(9,5)$ & $2(9,5)$ & \multicolumn{2}{|c|}{$17(81)$} \\
\hline $\begin{array}{l}\text { Toda informação necessária para decisões diagnósticas e terapêuticas está disponível } \\
\text { rotineiramente para mim. }\end{array}$ & $5(23,8)$ & $3(14,3)$ & \multicolumn{2}{|c|}{$13(61,9)$} \\
\hline Estagiários da minha profissão são adequadamente supervisionados. & $2(9,5)$ & $2(9,5)$ & \multicolumn{2}{|c|}{$17(81)$} \\
\hline Eu vivencio boa colaboração com os (as) enfermeiros (as) nesta área. & $2(9,5)$ & $1(4,8)$ & \multicolumn{2}{|c|}{$18(85,7)$} \\
\hline Itens isolados & $\begin{array}{l}\text { Discordo* } \\
\text { N (\%) }\end{array}$ & $\begin{array}{l}\text { Neutro } \\
\mathrm{N}(\%)\end{array}$ & \multicolumn{2}{|c|}{$\begin{array}{l}\text { Concordo* } \\
\mathrm{N}(\%)\end{array}$} \\
\hline Minhas sugestões sobre segurança seriam postas em ação se eu as expressasse à administração. & $2(9,5)$ & $3(14,3)$ & \multicolumn{2}{|c|}{$16(76,2)$} \\
\hline Eu vivencio boa colaboração com a equipe de médicos nesta área. & $3(14,3)$ & $2(9,5)$ & \multicolumn{2}{|c|}{$16(76,2)$} \\
\hline Falhas na comunicação que levam a atrasos no atendimento são comuns. ${ }^{* \star \star}$ & $5(23,8)$ & $1(4,8)$ & \multicolumn{2}{|c|}{$15(71,4)$} \\
\hline
\end{tabular}

*Parcial ou totalmente, ${ }^{* *}$ Não se aplica, ${ }^{* * *}$ Questão reversa. 
falhas funcionais podem contribuir para o estabelecimento de uma cultura positiva de segurança do paciente em qualquer serviço de saúde.

Portanto, ao considerar que os domínios do SAQ se mostraram em sua maioria com escore satisfatório, resta ressaltar a importância da realização de ações de educação em saúde sobre a segurança do paciente e gestão de riscos envoltos na assistência obstétrica e neonatal, tanto na maternidade estudada quanto nos demais serviços de saúde materno-infantil do país.

\section{AGRADECIMENTOS}

À direção e profissionais de saúde da maternidade Zacarias Júnior em Lagarto, Sergipe, Brasil.

\section{REFERÊNCIAS}

1. Rede Brasileira de Enfermagem e Segurança do Paciente. Estratégias para a segurança do paciente: manual para profissionais da saúde (REBRAENSP). Porto Alegre: EDIPUCRS, 2013.

2. Rigobello MCG, Carvalho RMFL, Cassiani SHB, Galon T, Capucho HC, Deus NN. Clima de segurança do paciente: percepção dos profissionais de enfermagem. Acta Paul Enferm. 2012;25(5):728-35 http://dx.doi.org/10.1590/S0103-21002012000500013

3. Quinto Neto A. Segurança dos pacientes, profissionais e organizações: um novo padrão de assistência à saúde. Rev Adm Saúde. 2006;8(33):153-8.

4. Taylor JA, Dominici F, Agnew J, Gerin D, Morlock L, Miller MR. Do nurse and patient injuries share common antecedents? An analysis of associations with safety climate and working conditions. BMJ Qual Saf. 2012;21(2):101-11. https://dx.doi.org/10.1136/bmjqs-2011-000082

5. Gabrani A, Hoxha A, Simaku A, Gabrani J. Research Application of the Safety Attitudes Questionnaire (SAQ) in Albanian hospitals: a cross-sectional study. BMJ Open. 2014;5(4). http://dx.doi.org/10.1136/bmjopen-2014-006528

6. Agência Nacional de Vigilância Sanitária (ANVISA). Serviços de atenção materna e neonatal: segurança e qualidade. Brasília: ANVISA, 2014

7. Coordenação Geral de Informações e Análise Epidemiológica (CGIAE). Consolidação Sistema de informações sobre nascidos vivos: 2011. Brasília: Ministério da Saúde, 2013.

8. DataSUS. Sistema de Informações Hospitalares do SUS $(\mathrm{SIH} /$ SUS). Disponível em: http://datasus.saude.gov.br/sistemas-eaplicativos/hospitalares/sihsus. Acesso em: 08 jan. 2018.

9. Barbetta PA. Estatística aplicada às ciências sociais. 7 ed. Florianópolis: UFSC, 2014.

10. Carvalho REFL, Cassiani SHB. Questionário Atitudes de Segurança: adaptação transcultural do Safety Attitudes Questionnaire - Short Form 2006 para o Brasil. Rev Latino-Am Enfermagem. 2012;20(3):575-82. http://dx.doi.org/10.1590/S0104-11692012000300020
11. Luiz RB, Simões ALA, Barichello E, Barbosa MH. Fatores associados ao clima de segurança do paciente em um hospital de ensino. Rev Latino-Am Enfermagem. 2015; 23(5):880-7. http://dx.doi.org/10.1590/0104-1169.0059.2627

12. Marinho MM, Radunz V, Barbosa SFF. Assessment of safety culture by surgical unit nursing teams. Texto Contexto Enferm. 2014;23(3):581-90. http://dx.doi.org/10.1590/0104-07072014002640012

13. Baratto MAM. Cultura de segurança do paciente: percepções e atitudes dos trabalhadores nas instituições hospitalares de Santa Maria. Dissertação (Mestrado) - Universidade Federal de Santa Maria. Santa Maria: 2015.

14. Carvalho REFL, Arruda LP, Nascimento NKP, Sampaio RL, Cavalcante MLSN, Costa ACP. Avaliação da Cultura de Segurança em Hospitais Públicos no Brasil. Rev Latino-Am Enfermagem. 2017;25:e2849.

http://dx.doi.org/10.1590/1518-8345.1600.2849

15. Carvalho PA, Göttems LBD, Pires MRGM, Oliveira MLC. Safety culture in the operating room of a public hospital in the perception of healthcare professionals. Rev Latino-Am Enfermagem. 2015;23(6):1041-8.

http://dx.doi.org/10.1590/0104-1169.0669.2647

16. Barbosa MH, Sousa EM, Félix MMS, Oliveira KF, Barichello E. Clima de segurança do paciente em um hospital especializado em oncologia. Rev Eletr Enf. 2015;17(4):1-9. https://dx.doi.org/10.5216/ree.v17i4.34614

17. Correia MM, Ferreira MM. Satisfação Profissional dos Enfermeiros. Dissertação (Mestrado) - Escola Superior de Enfermagem do Porto. Porto: 2016.

18. Relihan E, Glynn S, Daly D, Silke B, Ryder S. Measuring and benchmarking safety culture: application of the safety attitudes questionnaire to an acute medical admissions unit. Ir J Med Sci. 2009; 178(4):433-9.

https://dx.doi.org/10.1007/s11845-009-0352-2

19. World Health Organization (WHO). Human Factores in patint safety: review of topics and tools. Geneva: WHO, 2009. 